\title{
The observation of Gamma Ray Bursts and Terrestrial Gamma-ray Flashes with AGILE
}

E. Del Monte ${ }^{*, a}$, G. Barbiellinib ${ }^{\mathrm{b}, \mathrm{c}}$, F. Fuschino ${ }^{\mathrm{d}}$, A. Giuliani ${ }^{\mathrm{e}}$, F. Longo $^{\mathrm{b}, \mathrm{c}}$, M. Marisaldi $^{\mathrm{d}}$, S. Mereghetti $^{\mathrm{e}}$, E. Moretti $^{\mathrm{b}, \mathrm{c}}$, M. Trifoglio ${ }^{\mathrm{d}}$, G. Vianello ${ }^{\mathrm{e}}$, E. Costa ${ }^{\mathrm{a}}$, I. Donnarumma ${ }^{\mathrm{a}}$, Y. Evangelista ${ }^{\mathrm{a}, \mathrm{f}}$, M. Feroci $^{\mathrm{a}}$, M. Galli ${ }^{\mathrm{g}}$, I. Lapshov $^{\mathrm{a}}$,

F. Lazzarotto ${ }^{\mathrm{a}}$, P. Liparif ${ }^{\mathrm{f}}$, L. Pacciani ${ }^{\mathrm{a}}$, M. Rapisarda ${ }^{\mathrm{h}}$, P. Soffitta ${ }^{\mathrm{a}}$, M. Tavani ${ }^{\mathrm{a}, \mathrm{i}}$, S. Vercellone ${ }^{\mathrm{j}}$, S. Cutini ${ }^{\mathrm{k}}$,

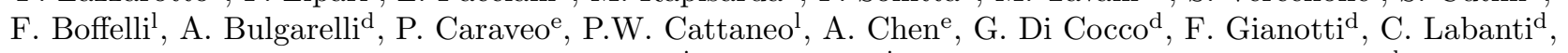

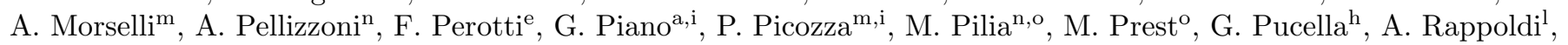
S. Sabatini ${ }^{\mathrm{a}, \mathrm{m}}$, E. Striani ${ }^{\mathrm{a}, \mathrm{m}}$, A. Trois ${ }^{\mathrm{a}}$, E. Vallazza ${ }^{\mathrm{b}}$, V. Vittorini ${ }^{\mathrm{a}}$, L. A. Antonelli ${ }^{\mathrm{p}, \mathrm{k}}$, C. Pittori ${ }^{\mathrm{k}}$, B. Preger ${ }^{\mathrm{k}}$, P. Santolamazza ${ }^{k}$, F. Verrecchiak ${ }^{k}$, P. Giommi ${ }^{q, k}$, L. Salotti ${ }^{\mathrm{q}}$

${ }^{a}$ INAF IASF Roma, Via Fosso del Cavaliere 100, I-00133 Roma, Italy ${ }^{b}$ INFN Trieste, Padriciano 99, I-34012 Trieste, Italy

${ }^{c}$ Dip. di Fisica, Università di Trieste, Via Valerio 2, I-34127 Trieste, Italy

${ }^{d}$ INAF IASF Bologna, Via Gobetti 101, I-40129 Bologna, Italy

${ }^{e}$ INAF IASF Milano, Via E. Bassini 15, I-20133 Milano, Italy

${ }^{f}$ Dip. di Fisica, Università di Roma "La Sapienza", P.le A. Moro 5, I-00185 Roma, Italy

${ }^{g}$ ENEA C. R. "E. Clementel", Via don Fiammelli 2, I-40128 Bologna, Italy

${ }^{h}$ ENEA C. R. Frascati, Via E. Fermi 45, I-00044 Frascati (Rm), Italy

${ }^{i}$ Dip. di Fisica, Univ. Roma Tor Vergata, Via della Ricerca Scientifica 1, I-00133 Roma, Italy

${ }^{j}$ INAF IASF Palermo, Via Ugo La Malfa 153, 90146 Palermo, Italy

${ }^{k}$ ASI Science Data Center, Via G. Galilei, I-00044 Frascati (Rm), Italy ${ }^{l}$ INFN Pavia, Via Bassi 6, I-27100 Pavia, Italy

${ }^{m}$ INFN Roma Tor Vergata, Via della Ricerca Scientifica, 1, I-00133 Roma, Italy

${ }^{n}$ INAF Osservatorio Astronomico di Cagliari, loc. Poggio dei Pini, strada 54, I-09012, Capoterra (Ca), Italy

${ }^{o}$ Dip. di Fisica e Matematica, Università dell'Insubria, Via Valleggio 11, I-20100 Como, Italy

${ }^{p}$ INAF Osservatorio Astronomico di Roma, Via di Frascati 33, I-00040 Monte Porzio Catone (Rm), Italy

${ }^{q}$ Agenzia Spaziale Italiana, Unità Segmento di Terra e Basi Operative, Viale Liegi 26, 00198 Roma, Italy

\section{Abstract}

Since its early phases of operation, the AGILE mission is successfully observing Gamma Ray Bursts (GRBs) in the hard X-ray band with the SuperAGILE imager and in the MeV range with the Mini-Calorimeter. Up to now, three firm GRB detections were obtained above $25 \mathrm{MeV}$ and some bursts were detected with lower statistical confidence in the 'same energy band. When a GRB is localized, either by SuperAGILE or Swift/BAT or INTEGRAL/IBIS or Fermi/GBM or IPN, inside the field of view of the Gamma Ray Imager of AGILE, a detection is searched for in the gamma ray band 'or an upper limit is provided. A promising result of AGILE is the detection of very short gamma ray transients, a few ms in duration and possibly identified with Terrestrial Gamma-ray Flashes. In this paper we show the current status of the observation of Gamma Ray Bursts and Terrestrial Gamma-ray Flashes with AGILE.

'Key words:

High energy astrophysics, Silicon microstrip detector, Gamma-ray burst, Terrestrial gamma-ray flashes

\section{Introduction}

The italian small satellite mission AGILE (see [1] for further information) has been designed, developed and produced having in mind the Gamma Ray Bursts (GRBs) as one of the most important scientific objectives. For this reason the X-ray monitor SuperAGILE (see [2] for a complete description of the instrument) and the nonimaging Mini-Calorimeter (described by [3]) are equipped

\footnotetext{
* Corresponding author

Email address: ettore.delmonte@iasf-roma.inaf.it (E. Del Monte)
}

with on-board trigger algorithms and dedicated telemetry data packets (see [4] and [5] for a complete description). Similar methods are used in the ground based analysis in order to detect GRBs.

At the time of writing, that is in two years of operations including the Commissioning and Science Verification Phase (until November 2007), the whole Cycle One (December 2007 - November 2008) and more than one half of the Cycle Two (December 2008 - November 2009), SuperAGILE localized 28 GRBs, about one per month. SuperAGILE is based on 1-D imaging and its field of view is composed of a central region, of $68^{\circ} \times 68^{\circ}$ in which both coordinates are encoded with an error radius of 3 , and 
four wings, where only 1-D images are produced as narrow strips of $6^{\prime} \times 68^{\circ}$. Out of $28 \mathrm{GRBs}, 17$ have an error radius of 3' while for the remaining 11 the position is given by the 1-D strip described above. In the same interval of time the Mini-Calorimeter (MCAL), a non imaging and all-sky scintillator detector, detected about one GRB per week in the energy band between $300 \mathrm{keV}$ and $100 \mathrm{MeV}$ (see 6] for the description of the MCAL GRB capabilities). During the same time span the AGILE Gamma Ray Imaging Detector (sensitive in the energy band between $30 \mathrm{MeV}$ and $30 \mathrm{GeV}$ and described by [1]) found three firm detections (GRB 080514B, GRB 090401B and GRB 090510) above $30 \mathrm{MeV}$ and two less significant ones (GRB 080721 and GRB 081001) in the same energy band.

\section{The observation of GRBs with AGILE}

Very little information is known about the emission of gamma rays from GRBs. In fact only a handful of events has been detected in the $30 \mathrm{MeV}-10 \mathrm{GeV}$ energy range by the Energetic Gamma Ray Experiment Telescope (EGRET) aboard the Compton Gamma Ray Observatory (CGRO) and the details are reported for example by [7] and references therein. The EGRET observations showed the possibility of an extended emission of gamma rays, longer than the prompt emission detected in the hard Xray band by the Burst And Transient Source Experiment (BATSE), including the extremely peculiar case of GRB 940217 ([8]), with a $18 \mathrm{GeV}$ photon detected after 1.3 hours from trigger.

The aim of efficiently observing GRBs in the gamma ray band has been a primary importance driver in the development of AGILE. In fact SuperAGILE and the Gamma Ray Imaging Detector (GRID) observe the same region of the Sky, although with fields of view of different dimensions, $\sim 1$ sr and $\sim 2.5$ sr respectively. Consequently each GRB localized by SuperAGILE is also in the center region of the GRID field of view, where the effective area and thus the sensitivity are around their maximum. In order to search for the detection of gamma rays, when a GRB is localized by SuperAGILE, the significance of the GRID events consistent with the burst time and position is estimated against the background. The procedure is applied to all the GRBs localized in the GRID field of view, for example by Swift/BAT, INTEGRAL/IBIS, Fermi/GBM or IPN and the details of the GRID data analysis in this task will be reported in a forthcoming paper.

The first GRB detected by AGILE above $30 \mathrm{MeV}$ is GRB 080514B (see [9] for the complete data analysis), that is also the first burst with a significant signal in the gamma ray band to be associated to an afterglow. The redshift of GRB 080514B has been measured with photometric methods at $z=1.8_{-0.3}^{+0.4}$ by [10]. The superposition of the SuperAGILE lightcurve and the arrival time and energy of the photons detected by the GRID is shown in fig. 1] From the plot it is clearly seen that the majority of the gamma rays (about $66 \%$ ) are emitted after the

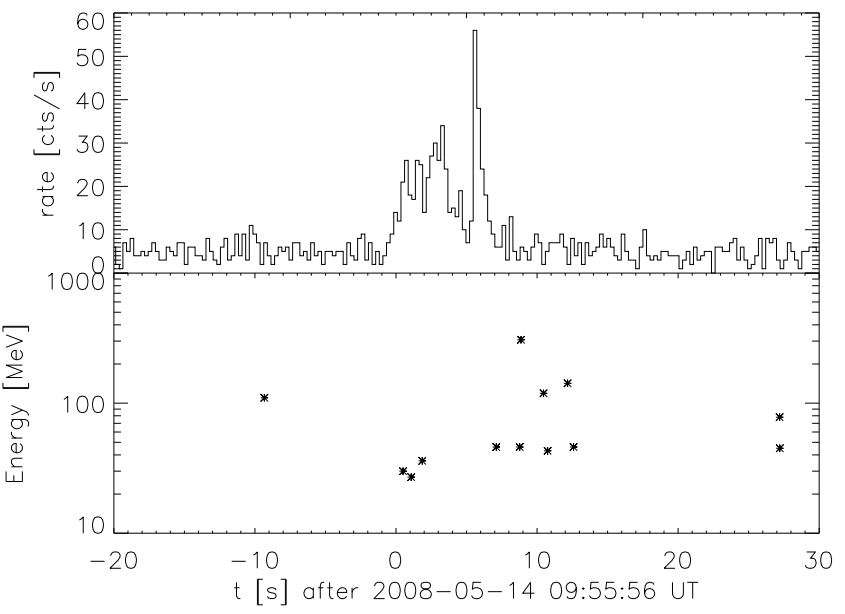

Figure 1: SuperAGILE lightcurve of GRB 080514B (upper panel) superimposed to the arrival time and energy of the gamma ray photons (lower panel).

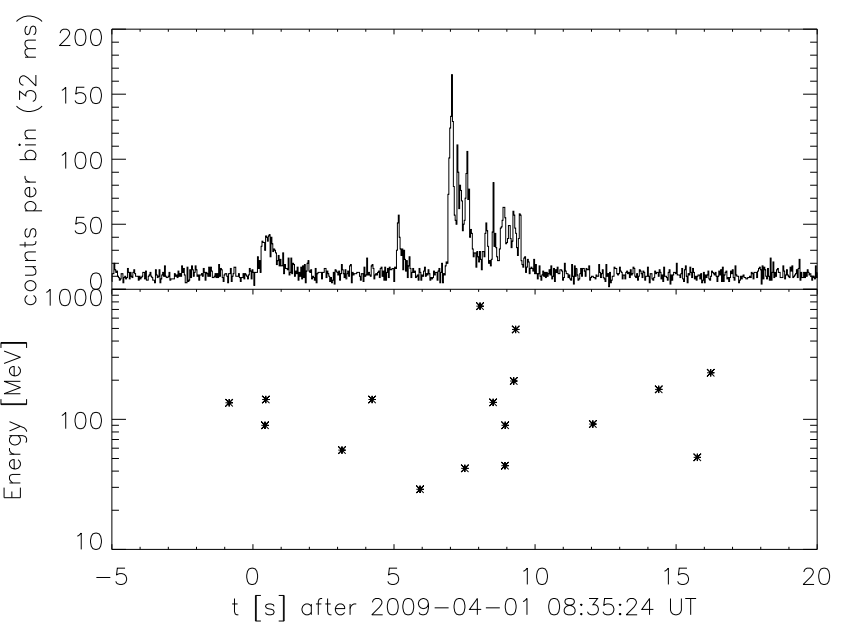

Figure 2: MCAL lightcurve of GRB 090401B (upper panel) superimposed to the arrival time and energy of the gamma ray photons (lower panel).

end of the prompt emission $(\mathrm{t} 0+7 \mathrm{~s})$ as detected in the hard X-ray band, while a minority of them (33\%) are emitted during the prompt emission. The fluence of GRB 080514B detected by the GRID between 20 and $50 \mathrm{MeV}$ is in good agreement with the extrapolation of the spectrum measured by Konus-Wind in the $20 \mathrm{keV}-5 \mathrm{MeV}$ energy range, fitted using a Band function (see [11]).

The prompt emission of GRB 090401B is characterized by a multipeak structure, detected up to $2.8 \mathrm{MeV}$ by MCAL (see fig. 2) and spanning about $10 \mathrm{~s}$. As shown in the figure, in this case the majority of the photons in gamma rays $(76 \%)$ is emitted simultaneously with the Xrays, while only a small fraction $(24 \%)$ is found after the end of the prompt phase.

The extended emission of gamma rays is particularly evident in the case of GRB 090510, that is also the first short GRB with a gamma ray emission detected by AGILE. As shown in fig. 3, the prompt emission is composed of a narrow and hard peak, of about $200 \mathrm{~ms}$ duration and 


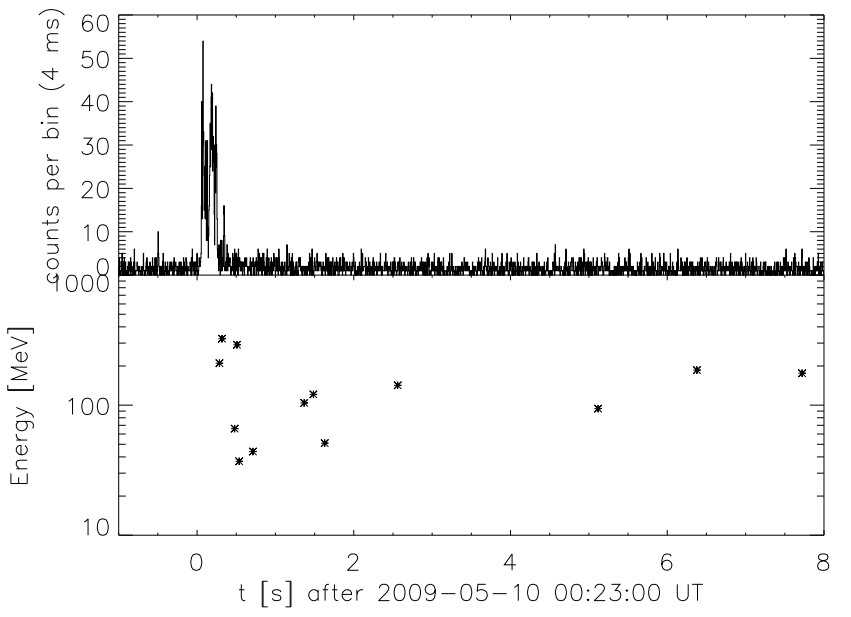

Figure 3: MCAL lightcurve of GRB 090510 (upper panel) superimposed to the arrival time and energy of the gamma ray photons (lower panel).

very clearly seen by MCAL up to tens of MeV energy. In this case the gamma rays are not simultaneous with the prompt emission while their detection starts just at the end (see fig. 3). GRB 090510 has been localized by Swift/BAT ([12]) and detected also by Fermi/LAT ([13]). The redshift has been measured spectroscopically by VLT/FORS2 and is $0.903([14])$.

Up to now AGILE has detected five GRBs in the gamma ray band in around two years of observation while the Fermi satellite mission has reported in the GCNs nine detections in about one year. If we sum the detection rate of the two missions we obtain a total rate of about one GRB per month in gamma rays. Since the two instruments, GRID and LAT, have similar fields of view, we may take into account the results of both satellites in order to obtain an overall detection rate. It seems that the phenomenon of the extended emission is a distinctive feature of the GRBs emitting in the gamma-ray band. In fact the same property has been detected, for example, by Fermi in the lightcurve of GRB 080916C ([15] ).

\section{Terrestrial Gamma-ray Flashes}

Terrestrial Gamma-ray Flashes (TGFs) are short and intense bursts with typical emission in the $\mathrm{MeV}$ region and duration of few ms, discovered by BATSE on CGRO (see [16]). The spectrum is harder than that of cosmic GRBs and the incoming direction is compatible with the Earth atmosphere. In nine years of operations BATSE detected only 78 TGFs mainly because of limitations in the trigger logic architecture. The sample statistics was greatly increased by RHESSI ([17]), whose first catalogue (see [18]) contains 820 TGFs detected between 2002 and 2008. The association between TGFs and atmospheric lightning and thunderstorm activity has been proven by [19] and [20] by means of temporal and spatial correlation with lighting strokes localized by their signature at VLF frequencies (sferics).

The observation of TGFs takes advantage of the MCAL on-board trigger logic, that is active on several timescales ranging between $285 \mu \mathrm{s}$ and $8 \mathrm{~s}$ and allows to send to telemetry photon-by-photon data in a time window of 60 s centered at the trigger time (including the energy information and a time tag with $1 \mu$ s accuracy) in case a trigger is issued. It is the first time that a timescale as short as $285 \mu \mathrm{s}$ is used in a space mission.

On the time scale of $16 \mathrm{~ms}$ or shorter, the average rate of the MCAL detections is 6.8 triggers per orbit, corresponding to about 95 triggers per day. In order not to miss any faint event, we decided to keep deliberately as low as possible the threshold of the on-board algorithm and to leave the event selection to the ground analysis, whose main purpose is the rejection of the triggers of instrumental origin.

A substantial fraction of the instrumental triggers is related to a particular payload status, clearly marked in the housekeeping data, and are easily rejected. After excluding this class we apply further criteria, based on the hardness ratio (HR) and fluence, in order to refine the dataset and reject instrumental triggers not identified in the previous step because of the limited timing accuracy of the housekeeping data. The HR is computed as the ratio between the number of events with energy higher than 1.4 $\mathrm{MeV}$ and the number of them below $1.4 \mathrm{MeV}$. The cut of $\mathrm{HR} \geq 0.5$ was finally selected and only the triggers with at least 10 photons in the burst time interval are considered. All the events satisfying the criteria listed above are visually inspected to exclude further contamination from instrumental effects and properly check the start time and duration of the events. Following these criteria, a total number of 34 events are found between June 2008 and March 2009 (corresponding to a rate of about 4 events per month), which are considered as good TGF candidates.

The geographical coordinates of each TGF are derived from the footprint of AGILE at the trigger time. Since AGILE is on an equatorial orbit with $\sim 2.5^{\circ}$ of inclination, only a very narrow region across the Equator is span and no high latitude coverage can be obtained. The geographical distribution of the MCAL dataset is shown in fig. 4. In the figure the clustering of the TGFs above Africa, with about half of the events ranging in the longitude interval from $0^{\circ}$ to $30^{\circ}$, and South-East Asia, with about one third of the triggers in the interval between $90^{\circ}$ and $120^{\circ}$, is clearly seen. The AGILE TGFs geographical and local time distributions well match those for the RHESSI TGFs, when a consistent cut in latitude is applied.

The sum of the net exposure of all the TGFs in the MCAL sample amounts to $51 \mathrm{~ms}$. The dataset shows a total number of 47 photons with energy higher than $10 \mathrm{MeV}$ and 8 photons above $20 \mathrm{MeV}$. These number are highly significant since, in the same energy intervals, the expected background counts are 2.3 and 1.3 respectively. The highest photon's energy detected is larger than $40 \mathrm{MeV}$. The cumulative spectrum can be well fitted with 


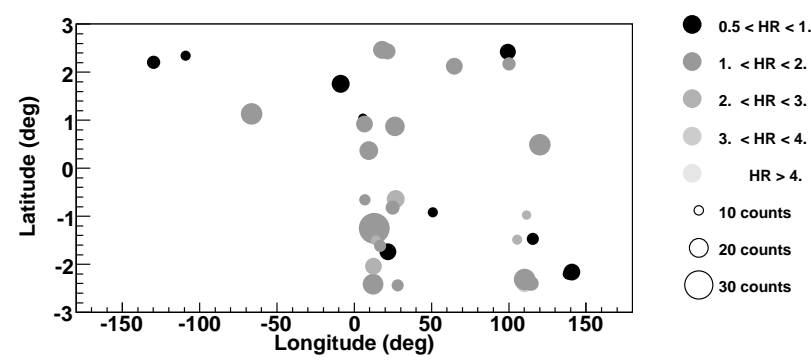

Figure 4: Geographical distribution of the AGILE TGF sample detected between June 2008 and March 2009. For each event the greyscale indicates the $\mathrm{HR}$ and the marker size is proportional to the number of detected counts.

a power law with exponential cutoff, with a cutoff energy of about $9 \mathrm{MeV}$. The same spectral model closely match also the RHESSI cumulative spectrum, with a difference in the normalization factor of about a factor 2 . This difference in normalization may be ascribed both to calibration issues and to dead time effects. The effects of dead time were proven to be of particular importance for RHESSI and BATSE as shown in [21] and are still under evaluation for what concerns MCAL. Ref. 22] reports a detailed description of the selection criteria and the TGF candidate properties, as well as the comparison of the AGILE sample with the RHESSI one.

\section{Discussion and conclusions}

The observation of GRBs by Fermi and AGILE missions is showing that the emission of gamma rays from this class of sources is rather uncommon, with a detection rate of about one event per month. The continuing observation will allow to increase the statistic of the data sample and may help to find correlations between the detection of gamma rays and other features of the GRBs. So far the gamma rays are detected mainly during the prompt phase of the GRBs and usually belong to an extended emission, lasting longer than the prompt emission in hard X-rays. The fraction of gamma rays in the extended emission may vary, depending on the position of the peaks in the lightcurve. Another important property, reported in the case of GRB $080514 \mathrm{~B}$, is that the fluence in gamma rays is found on the extrapolation of the spectrum of the prompt emission detected up to the $\mathrm{MeV}$ region. A similar feature has been found in the spectrum of GRB 080916C, detected by Fermi/LAT ([15]). The analysis of the energetics of other gamma ray emitting bursts is still in progress. Up to now no peculiar feature is found in the X-ray afterglow of gamma ray emitting GRBs but the sample is still small.

After the onset of the timescale shorter than $64 \mathrm{~ms}$ on the on-board trigger of the AGILE MCAL, the instrument is detecting a population of short and intense bursts with properties compatible with those of TGFs. Both the geographical and local time distributions as well as the cumulative spectrum of the AGILE TGF sample well match the same distributions for the RHESSI sample, confirming that AGILE is actually detecting TGFs and the goodness of the selection criteria applied. Improvements in the selection strategy and trigger logic are expected to increase the TGF detection rate. Thanks to the almost equatorial orbit, AGILE can provide a continuous monitoring of the equatorial region, especially concerning central Africa and South East Asia, where some of the most severe TGFproducing thunderstorms develop.

\section{Acknowledgments}

AGILE is a mission of the Italian Space Agency, with co-participation of INAF (Istituto Nazionale di Astrofisica) and INFN (Istituto Nazionale di Fisica Nucleare). This work was partially supported by ASI grants I/R/045/04, I/089/06/0, I/011/07/0 and by the Italian Ministry of University and Research (PRIN 2005025417). INAF personnel at ASDC are under ASI contract I/024/05/1. The authors warmly acknowledge the support by the team of the InterPlanetary Network (IPN).

\section{References}

[1] M. Tavani et al. Nucl. Instr. and Meth. in Phys. Res. A, 588, 52 (2008)

[2] M. Feroci et al., Nucl. Instr. and Meth. A, 581, 724 (2007)

[3] C. Labanti et al., Nucl. Instr. and Meth. A, 598, 470-479 (2009)

[4] E. Del Monte et al., Proceedings of the 5th SCINEGHE Workshop, 2007, arXiv:0712.1548

[5] F. Fuschino et al., Nucl. Instr. and Meth. A, 588, 17-21 (2008)

[6] M. Marisaldi et al., A\&A, 490, 1151 (2008)

[7] B. Dingus, AIP Conf. Ser., 558, 383 (2001)

[8] K. Hurley, et al., Nature, 372, 652 (1994)

[9] A. Giuliani et al., A\&A, 491, L25-L28 (2008)

[10] A. Rossi et al., A\&A, 491, L29-L32 (2008)

[11] S. Golenetskii et al., GCN 7751 (2008)

[12] E. A. Hoversten et al., GCN 9331 (2009)

[13] M. Ohno et al., GCN 9334 (2009)

[14] A. Rau et al., GCN 9353 (2009)

[15] A. Abdo et al., Science, 323, 1688 (2009)

[16] G. J. Fishman et al., Science, 164, 1313 (1994)

[17] D. M. Smith et al., Science 307, 1085 (2005)

[18] B. W. Grefenstette et al., J. Geophys. Res., 114, A02314 (2009)

[19] U. S. Inan et al., Geophysical Research Letters, 23, 1017-1020 (1996)

[20] M. B. Cohen et al., Journal of Geoph. Res., 111 (2006)

[21] B. W. Grefenstette et al., Geophysical Research Letters, 35 (2008)

[22] M. Marisaldi et al., J. Geophys. Res. 115 (2010) A00E13. 